\title{
Effects of pentobarbital on fixed-ratio discrimination*
}

\author{
JORDAN ROSENBERG and JAMES H. WOODS \\ University of Michigan, Ann Arbor, Michigan 48104
}

\begin{abstract}
Doses of pentobarbital in the range $5-15 \mathrm{mg} / \mathrm{kg}$ were administered to pigeons trained to respond under a fixed-ratio discrimination procedure. Pentobarbital administration produced a dose-related reduction in the level of sensitivity of the discrimination between the two ratios.
\end{abstract}

Rilling and McDiarmid (1965) trained pigeons to discriminate 35 responses from 50 responses in a situation where one or the other number of responses was required on a center key (a fixed-ratio requirement) in order to produce the opportunity to respond to either one of two side keys. Responses to the side keys were differentially reinforced according to the number of responses previously emitted on the center key. Analysis of the data by the methods of signal detection theory (Green \& Swets, 1966) showed that the ratio values of 35 and 50 were nearly maximally detectable. When the smaller ratio was increased in several steps, thereby reducing the difference between the two ratios, the data showed corresponding reductions in the level of detectability.

Branch (1974) used this ratio discrimination procedure to establish a behavioral baseline in a study that assessed the effect of pentobarbital administration upon discrimination accuracy. The measure of discrimination accuracy employed by Branch was "percent correct" which is defined as the ratio of the number of reinforced side key responses to the total number of side key responses. When pentobarbital was administered in doses ranging from $2-8 \mathrm{mg}$ (Approximately $4-16 \mathrm{mg} / \mathrm{kg}$ ), a dose-related reduction in discrimination accuracy was observed. The analysis failed, however, to distinguish between those changes in percent correct that resulted from changes in sensitivity to the fixed-ratio values and those changes in percent correct that resulted from changes in response bias. The virture of the methods of analysis of signal detection theory as applied by Rilling and McDiarmid (1965) is that they permit such a distinction to be made.

The study reported here also examined the effect of pentobarbital administration upon performance in the ratio discrimination procedure. The effects of pentobarbital administration upon both sensitivity to the fixed-ratio values and response bias were determined.

\section{METHOD}

\section{Subjects}

The subjects were three adult make White Carneaux pigeons

*This research was supported in part by United States Public Health Service Grant DA-00154. Reprints may be obtained from Jordan Rosenberg, Department of Psychology, California State University, Hayward, Hayward, Califonia 94542 .
$(008,012,013)$, experimentally naive, maintained at $80 \%$ of their free feeding weights.

\section{Apparatus}

The apparatus consisted of a standard operant conditioning chamber with three response keys and grain feeder. Experimental events were controlled with electromechanical programming units, and data were recorded on impulse counters.

\section{Procedure}

The procedure followed the yes-no paradigm of signal detection theory (Green \& Swets, 1966) and was similar to that described by Rilling and McDiarmid (1965) and Branch (1974).

Each trial began with the center key lighted and the side keys darkened. The subject responded until the center key was darkened and, simultaneously, the side keys were lighted. The number of responses required to darken the center key was either 10 (the signal ratio) or 50 (the noise ratio). A single response to a lighted side key was followed by either reinforcement (a 4-sec presentation of grain) or $60 \mathrm{sec}$ of blackout. A response to the left side key (a yes response) was reinforced if, and only if, it followed the signal ratio. A response to the right side key (a no response) was reinforced if, and only if, it followed the noise ratio. All other responses to the side keys were followed by a $60-\mathrm{sec}$ blackout. When the reinforcement or blackout period terminated, a new trial began.

The value of the ratio selected for each trial was chosen randomly, and the two ratios were equally likely to be selected. Daily sessions lasted for 55 trials with data recorded from the last 50 trials. The data recorded from each session were the number of side key responses accumulated separately in four categories according to key and according to the value of the preceding ratio.

The subjects were trained by a successive approximation procedure. When the ratio values of 10 and 50 were reached, they remained in effect until there were essentially no blackouts and until this performance appeared stable. These data then served as the basis for assessing the effects of pentobarbital administration.

A solution of pentobarbital in distilled water was administered by intramuscular injection once per week. The dose of pentobarbital administered was varied and took on the values 5, $7.5,10$, and $15 \mathrm{mg} / \mathrm{kg}$ as the salt. Volume of solution injections were given according to $0.1 \mathrm{cc} / 100 \mathrm{~g}$ of body weight, and dose was varied by concentration change. Each dose was administered several times as indicated by the number of points in Figure 1, each of which represents a single session. Saline was administered as a control 1 or 2 days prior to each drug day.

\section{RESULTS}

The-distribution of side key responses was analyzed to determine the effect of pentobarbital administration on the subjects' sensitivity and response bias. The analysis 
required that the probability of a yes response (R1) be calculated twice, once for trials on which the signal ratio (S1) had been presented and once again for trials on which the noise ratio (SO) had been presented. In the terminology of signal detection theory the former value, $\mathrm{p}(\mathrm{R} 1 / \mathrm{S} 1)$, is the hit rate and the latter value, $\mathrm{p}(\mathrm{R} 1 / \mathrm{S} 0)$, is the false alarm rate. The hit rate has been plotted as a function of the false alarm rate in Figure 1.

Each point in Figure 1 comes from a single session and all drug sessions are represented. However, data obtained from saline control sessions have been omitted. The majority of these sessions were represented by the point $(0,1)$ and the remainder were very close to this point.

The point $(0,1)$ represents the level of greatest sensitivity because it is obtained when the subject always responds yes to the signal and never responds yes to the noise. On the other hand, points along the positive diagonal are obtained when the probability of responding yes is the same for both signal and noise trials, a sensitivity level of zero. The extremes of response bias occur when the subject always responds yes or always responds no on both signal and noise trials. These performances are represented by the points $(1,1)$ or $(0,0)$, respectively. The negative diagonal represents unbiased performance.

The data plotted in Fig. 1 show considerable variability. This variability appears in both the level of sensitivity and the amount of bias. A portion of the variability may be due to the small number of trials involved. Each point was based on 50 trials, each coordinate value on 25 . Despite the variability, inspection of Figure 1 reveals a reduction in the level of sensitivity as the dose of pentobarbital administered increased, a finding confirmed by additional data presented below. The effect of pentobarbital administration on bias is less clear. For Subject 008, the intermediate dose produced bias in one direction and the largest dose produced bias in the other direction. For Subjects 012 and 013 bias occurred in different directions in different sessions. It should be noted that the two variables that are usually manipulated experimentally to produce changes in bias, the proportion of trials on which the signal is presented and the consequences of yes and no responses, were held constant here.

Quantitative indices of sensitivity and bias were calculated from the recorded data according to the procedures suggested by Smith (1974) and are presented in Table 1 . These indices are approximately proportional to the values of $\left(d^{\prime}\right)$ and $(\ln \beta)$, respectively, of signal detection theory. For each subject the doses of pentobarbital administered are presented in the order of decreasing sensitivity and again in the order of decreasing bias. It should be noted that the bias index was positive when the response bias favored no responses and was negative when the response bias favored yes
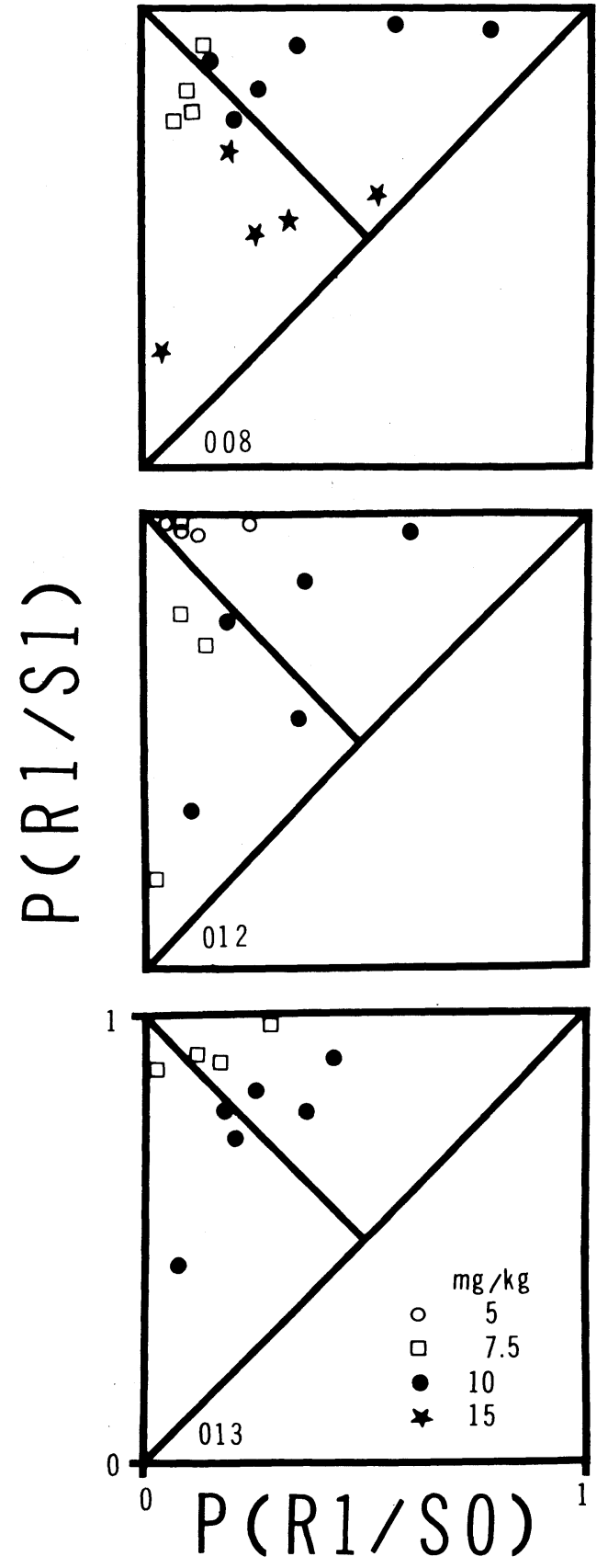

Fig. 1. The hit rate, $p(R 1 / S 1)$, plotted as a function of the false alarm rate, $p(R 1 / S 0)$, where $R 1$ is a response to the left side key and where $S 1$ and $S 0$ are the ratio values 10 and 50 , respectively. Data are presented for all subjects $(008,012,013)$ and from all experimental sessions during which pentobarbital was administered.

responses. The data of Table 1 show that as the dose of pentobarbital administered increased the sensitivity of the subject decreased. On a few occasions, however, the intermediate dose of pentobarbital was found to have either an exceptionally strong or an exceptionally weak effect on sensitivity. There does not appear to be any 
Table 1

Resulting Values of the Sensitivity Index and the Bias Index for Each Subject and Each Dose of Pentobarbital Administered

\begin{tabular}{|c|c|c|c|c|}
\hline & Dose & Sensitivity & Dose & Bias \\
\hline Subject 008 & $\begin{array}{c}7.5 \\
10 \\
7.5 \\
7.5 \\
7.5 \\
10 \\
10 \\
10 \\
10 \\
15 \\
10 \\
15 \\
15 \\
15 \\
15\end{array}$ & $\begin{array}{r}4.10 \\
3.65 \\
3.57 \\
3.46 \\
3.06 \\
2.83 \\
2.75 \\
2.50 \\
2.41 \\
2.13 \\
1.59 \\
1.53 \\
1.15 \\
.84 \\
.19\end{array}$ & $\begin{array}{c}15 \\
7.5 \\
7.5 \\
7.5 \\
15 \\
15 \\
15 \\
10 \\
15 \\
10 \\
10 \\
7.5 \\
10 \\
10 \\
10\end{array}$ & $\begin{array}{r}1.15 \\
.86 \\
.49 \\
.48 \\
.31 \\
.25 \\
.14 \\
.06 \\
-.02 \\
-.17 \\
-.27 \\
-.29 \\
-.97 \\
-1.17 \\
-1.66\end{array}$ \\
\hline Subject 012 & $\begin{array}{l}5 \\
7.5 \\
5 \\
5 \\
5 \\
7.5 \\
7.5 \\
10 \\
10 \\
7.5 \\
10 \\
10 \\
10\end{array}$ & $\begin{array}{r}7.86 \\
6.17 \\
5.08 \\
5.03 \\
4.69 \\
3.44 \\
2.62 \\
2.48 \\
2.47 \\
2.44 \\
2.05 \\
1.46 \\
.82\end{array}$ & $\begin{array}{c}7.5 \\
10 \\
7.5 \\
7.5 \\
10 \\
10 \\
5 \\
10 \\
5 \\
5 \\
7.5 \\
10 \\
5\end{array}$ & $\begin{array}{r}2.18 \\
.86 \\
.71 \\
.44 \\
.15 \\
.08 \\
.00 \\
-.33 \\
-.46 \\
-1.05 \\
-1.51 \\
-1.51 \\
-2.30\end{array}$ \\
\hline Subject 013 & $\begin{array}{c}7.5 \\
7.5 \\
7.5 \\
7.5 \\
10 \\
10 \\
10 \\
10 \\
10 \\
10 \\
\end{array}$ & $\begin{array}{l}5.88 \\
4.99 \\
4.83 \\
3.77 \\
2.67 \\
2.63 \\
2.60 \\
2.39 \\
2.29 \\
1.87 \\
\end{array}$ & $\begin{array}{c}7.5 \\
10 \\
10 \\
10 \\
10 \\
7.5 \\
10 \\
7.5 \\
10 \\
7.5\end{array}$ & $\begin{array}{r}1.76 \\
1.25 \\
.25 \\
.07 \\
-.22 \\
-.24 \\
-.33 \\
-. .37 \\
-1.02 \\
-2.41\end{array}$ \\
\hline
\end{tabular}

Note-For the first pair of columns the doses are presented in the order given by the values of the sensitivity index. For the second pair of columns the doses are presented in the order given by the values of the bias index. It should be noted that the bias index was positive when the response bias favored "no" responses and was negative when the response bias favored "yes" responses. systematic relationship between dose of pentobarbital administered and response bias.

\section{DISCUSSION}

The main finding reported here is the functional relation between the dose of pentobarbital administered and the resulting level of sensitivity obtained under the fixed-ratio discrimination procedure. A similar finding was obtained by Rilling and McDiarmid (1965) in a study using the fixed-ratio discrimination procedure in which the independent variable was the size of the smaller ratio value with the larger ratio value held constant. In their study and in the study reported here a further finding was that changes in the independent variable did not produce consistent changes in the amount of response bias obtained. The response bias effects may, as Rilling and McDiarmid (1965) point out, result from the relatively greater control exerted by other independent variables as the relative con trol exerted by the ratio values is lessened.

Branch reported that administration of pentobarbital reduced discrimination accuracy obtained under the fixed-ratio discrimination procedure in a dose-related way. This reduction in discrimination accuracy could reflect a reduction in sensitivity or a change in response bias or both. To this extent, Branch's (1974) analysis of the effect of pentobarbital administration on discrimination performance is incomplete. The findings reported here confirm the previous findings and further establish that administration of pentobarbital results in a dose-proportional reduction in the level of sensitivity obtained under the fixed-ratio discrimination procedure.

\section{REFERENCES}

Branch, M. N. Behavior as a stimulus: Joint effects of d-amphetamine and pentobarbital. The Journal of Pharmacology and Experimental Therapeutics, 1974, 189, $33-41$.

Green, D. M., and Swets, J. A. Signal detection theory and psychophysics. John Wiley and Sons, New York, 1966.

Rilling, M., \& McDiarmid, C. Signal detection in fixed-ratio schedules, Science, 1965, 148, 526-527.

Smith, J. E. K. Some simplified indices of sensitivity and bias. Michigan Mathematical Psychology Program, technical report,
1974-1975, No. 5.

(Received for publication September 3, 1974.) 